\title{
MHD Powell-Eyring fluid flow with non-linear radiation and variable thermal conductivity over a permeable cylinder
}

\author{
Amit Parmar*, Shalini Jain \\ Dept. of Mathematics \& Statistics, Manipal University Jaipur, Jaipur-303007, Rajasthan, India
}

Corresponding Author Email: amit.198631@gmail.com

https://doi.org/10.18280/ijht.360108

Received: 4 September 2017

Accepted: 3 January 2018

\section{Keywords:}

non-linear radiation, non-linear heat source, variable thermal conductivity, Powell-Eyring fluid

\begin{abstract}
In this article, we have investigated the influence of magneto-hydro-dynamic (MHD) Powell-Eyring fluid flow in the presence of non-linear radiation, space dependent internal heat source and variable thermal conductivity over a permeable cylinder with suction/injection effects. We have considered Soret, Dufour and non-linear chemical reaction effect on heat and concentration equations. Using similarly transformation, the governing PDEs are changed into non-linear coupled ODEs and solved by R-K forth order with shooting method. The impact of various parameters such as Powell- Eyring fluid parameters $(\mathrm{K})$, Dufour parameter $(\mathrm{Du})$, radiation parameter $(\mathrm{R})$, small scale parameter $\left({ }^{\varepsilon}\right)$, Prandtl number $(\mathrm{Pr})$, curvature parameter $(\gamma)$, Schmidt number $(\mathrm{Sc})$, chemical reaction parameter $(\mathrm{Kn})$, Eckert number $(\mathrm{Ec})$, relative temperature ratio parameter $\left({ }^{\theta_{w}}\right)$, Soret parameter $(\mathrm{Su})$, magnetic field parameter $(\mathrm{M})$ and $\left(\mathrm{A}^{*}\right)$ and $\left(\mathrm{B}^{*}\right)$ are specific and temperature heat source on axial momentum, heat and concentration profiles have been analyzed graphically and skin friction coefficient, local Nusselt number and local Sherwood number can be discussed tabulated.
\end{abstract}

\section{INTRODUCTION}

Non-Newtonian fluids have greatest importance role in the theory of fluid mechanics. Fluids which shear stress and shear rate are non-linear called non -Newtonian fluid. Tooth paste, food oil, blood etc. are non-Newtonian fluids. The PowellEyring model, firstly in the phenomena of power -law model, it is assumed from kinematic theory of liquid rather than the experiential relation. Khan et al. [1] investigated MHD fluid flow with variable properties. Krishna et al. [2] studied unsteady Powell-Eyring fluid flow past an inclined stretching sheet. Mahanthesh et al. [3] examined unsteady 3-D MHD Eyring-Powell fluid past a convectively heated stretching sheet. Several researchers investigated (Akbar et al. [4], Javed et al [5], Hayat et al. [6-10] and Gaffar et al. [11]) 2D and 3D flow for MHD and radiative Powell-Eyring fluid towards a stretching sheet with various boundary condition.

Heat transfer phenomena has significant applications of plastic sheets, spinning of fibers, polymer, plasma studies, MHD power generator, petroleum industries, cooling of nuclear reactors, glass fiber production and paper production etc. Radiation effects may be important role in controlling heat transfer.

MHD (magneto-hydro-dynamic) is the study of the magnetic properties of electrically conducting fluids. Magneto-fluids include salt water or electrolytes, liquid metals and plasmas. Several researchers (Madhu et al. [12], Makinde [13], Jain et al. [14-18], Dasa et al. [19] and Chauhan et al. [20-22]) proposed heat transfer phenomena for the impact of various physical condition with various geometries and surface conditions.
Keeping all these specifics in mind, we intend to study the boundary layer flow and heat transfer of MHD Powell-Eyring fluid flow in the presence of non-linear radiation, space dependent internal heat source and variable thermal conductivity over a permeable cylinder with suction/injection effects. We have considered Soret, Dufour and non-linear chemical reaction effect on heat and concentration equations.

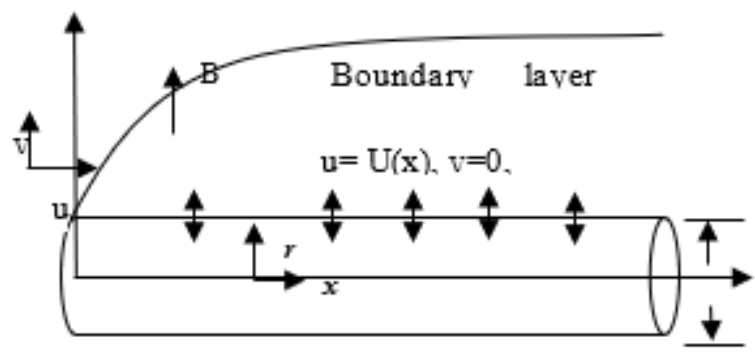

Figure 1. Physical diagram of the problem

\section{MATHEMATICAL FORMULATION}

2D boundary layer flow for Power-Eyring fluid over a permeable cylinder is considered. The axis of the cylinder is taken along the $x$ axis and $r$ is taken along radial direction. The coordinate system and flow regime is illustrated as exposed in the Figure 1. The Cauchy stress tensor in PowerEyring fluid is given by 
$\tau_{i j}=\mu \frac{\partial u_{i}}{\partial x_{j}}+\frac{1}{\beta} \sinh ^{-1}\left(\frac{1}{\gamma} \frac{\partial u_{i}}{\partial x_{j}}\right)$

where $\mu$ : viscosity coefficient, $\beta$ and $\gamma$ : material fluid parameters. The governing equations can be written as Khan et.al [1].

$\frac{\partial(r u)}{\partial x}+\frac{\partial(r v)}{\partial r}=0$

$u \frac{\partial u}{\partial x}+v \frac{\partial u}{\partial r}=U_{e} \frac{\partial U_{e}}{\partial x}+\left(v+\frac{1}{\rho \beta c}\right) \frac{\partial^{2} u}{\partial r^{2}}-\frac{1}{2 \rho \beta c^{3}}\left(\frac{\partial u}{\partial r}\right)^{2} \frac{\partial^{2} u}{\partial r^{2}}$

$+\frac{1}{r}\left(v+\frac{1}{\rho \beta c}\right) \frac{\partial u}{\partial r}-\frac{1}{6 r \rho \beta c^{3}}\left(\frac{\partial u}{\partial r}\right)^{3}-\frac{\sigma B_{0}^{2}}{\rho}\left(u-U_{e}\right)$

$u \frac{\partial T}{\partial x}+v \frac{\partial T}{\partial r}=\frac{1}{r \rho C_{p}} \frac{\partial}{\partial r}\left(k(T) r \frac{\partial T}{\partial r}\right)-\frac{1}{\rho C_{p}}\left(\frac{\partial q_{r}}{\partial r}\right)$

$+\bar{D}\left(\frac{\partial^{2} C}{\partial r^{2}}+\frac{1}{r} \frac{\partial C}{\partial r}\right)+\frac{q^{\prime \prime \prime}}{\rho C_{p}}$

$u \frac{\partial C}{\partial x}+v \frac{\partial C}{\partial r}=D_{m}\left(\frac{\partial^{2} C}{\partial r^{2}}+\frac{1}{r} \frac{\partial C}{\partial r}\right)+\bar{S}\left(\frac{\partial^{2} T}{\partial r^{2}}+\frac{1}{r} \frac{\partial T}{\partial r}\right)-k_{n}\left(C-C_{\infty}\right)^{n}$

The boundary conditions are

$u(x, a)=U_{w}, v(x, a)=-V_{w}, T(x, a)=T_{w}=T_{\infty}+T_{0} \frac{x}{l}$,

$C(x, a)=C_{w}=C_{\infty}+C_{0} \frac{x}{l}$ at $r=a$

$u(x, a) \rightarrow U_{e}, T(x, a) \rightarrow T_{\infty}, C(x, a) \rightarrow C_{\infty} \quad$ at $r \rightarrow \infty$

where $U_{w}=\frac{x U_{0}}{l}, U_{e}=\frac{x U_{\infty}}{l}$,

Rosseland approximation can be considered, the radiative heat flux, $q_{r}$ becomes (Ska et.al [23]) $\frac{\partial q_{r}}{\partial y}=\frac{\partial}{\partial y}\left(\frac{-4 \sigma}{3 k^{*}} \frac{\partial T^{4}}{\partial y}\right)$

$\sigma$ : Stefan-Boltzmann constant: $k^{*}$ absorption coefficient and the equation (4) become

$u \frac{\partial T}{\partial x}+v \frac{\partial T}{\partial r}=\frac{1}{\rho C_{p}} \frac{\partial}{\partial r}\left(k(T) r \frac{\partial T}{\partial r}\right)+\bar{D}\left(\frac{\partial^{2} C}{\partial r^{2}}+\frac{1}{r} \frac{\partial C}{\partial r}\right)$

$+\frac{k_{\infty} U_{w}}{\rho C_{p} x V}\left[A^{*} f^{\prime}\left(T_{s}-T_{\infty}\right)+B^{*}\left(T-T_{\infty}\right)\right]+\frac{1}{\rho C_{p}} \frac{16 \sigma}{3 k^{*}} \frac{\partial^{2} T^{4}}{\partial y^{2}}$

where t: time, $C_{p}$ : Specific heat, $K(T)=k_{\infty}(1+\varepsilon \theta)$ : thermal conductivity depending of temperature (ref. [24]) $\rho$ : fluid density, T: fluid temperature, C: fluid concentration.

The similarity transformations (Khan et.al [1]) as given below are introduced:
$u=\frac{U_{0} x}{l} f^{\prime}(\eta) \quad, \quad v=-\frac{a}{r} \sqrt{\frac{v U_{0}}{l}} f(\eta) \quad, \quad \theta(\eta)=\frac{T-T_{\infty}}{T_{s}-T_{\infty}}$,

$\eta=\frac{r^{2}-a^{2}}{2 a} \sqrt{\frac{U_{0}}{v l}}$ and $\phi(\eta)=\frac{C-C_{\infty}}{C_{s}-C_{\infty}}$

$2(1+K) \gamma f^{\prime \prime}-f^{\prime 2}+(1+K)(1+2 \gamma \eta) f^{\prime \prime}+A^{2}+f f^{\prime \prime}-$

$\frac{4}{3} K \lambda_{2} \gamma(1+2 \gamma \eta) f^{\prime \prime 3}-K \lambda_{2}(1+2 \gamma \eta)^{2} f^{\prime \prime \prime} f^{\prime \prime 2}-M\left(f^{\prime}-A\right)=0$

$2(1+\varepsilon \theta) \gamma \theta^{\prime}-\operatorname{Pr}\left(\theta f^{\prime}-f \theta^{\prime}\right)+(1+2 \gamma \eta)(1+\varepsilon \theta) \theta^{\prime \prime}$

$+(1+2 \gamma \eta) \varepsilon \theta^{\prime 2}+\frac{4 R}{3}\left(\left(\theta_{w}-1\right) \theta+1\right)^{3} \gamma \theta^{\prime}+A^{*} f^{\prime}+B^{*} \theta$

$+\frac{4 R}{3}\left(\left(\theta_{w}-1\right) \theta+1\right)^{3}(1+2 \gamma \eta) \theta^{\prime \prime}+\operatorname{Pr} D u\left(2 \gamma \phi^{\prime}+(1+2 \gamma \eta) \phi^{\prime \prime}\right)$

$+4 R\left(\theta_{w}-1\right)\left(\left(\theta_{w}-1\right) \theta+1\right)^{2}(1+2 \gamma \eta) \theta^{12}=0$

$\phi^{\prime \prime}(1+2 \gamma \eta)+2 \phi^{\prime} \gamma+\operatorname{Su} \operatorname{Le}\left((1+2 \gamma \eta) \theta^{\prime \prime}+\theta \gamma\right)$

$-L e\left(f^{\prime} \phi-f \phi^{\prime}\right)-L e K_{n} \phi^{n}=0$

Boundary conditions are following as:

$$
\begin{array}{lll}
f=S, f^{\prime}=1, \theta=1, \phi=1 & \text { at } & \eta=0 \\
f^{\prime} \rightarrow A, \theta \rightarrow 0, \phi \rightarrow 0 & \text { at } & \eta \rightarrow \infty
\end{array}
$$

where prime denotes with respect to $\eta, f$ : dimensionless stream function, $\theta$ : dimensionless temperature $\phi$ : dimensionless concentration, $K=\frac{1}{\mu \beta \gamma}$ : material fluid parameters, $\lambda_{2}=\frac{U_{0}^{3} x^{2}}{2 l^{3} c^{2} v}$ : dimensionless fluid parameter, $\gamma=\sqrt{\frac{v l}{U_{0} a^{2}}}:$ curvature parameter, $R=\frac{4 \sigma T_{\infty}^{3}}{k^{*} k_{\infty}}:$ radiation parameter, $S=\frac{r V_{w}}{a} \sqrt{\frac{l}{v U_{0}}}:$ suction /injection parameter, $D u=\frac{\bar{D}}{v}\left(\frac{C_{w}-C_{\infty}}{T_{w}-T_{\infty}}\right)$ : Dufour number, $S u=\frac{\bar{S}}{v}\left(\frac{T_{w}-T_{\infty}}{C_{w}-C_{\infty}}\right):$ Soret number, $k_{\infty}$ : thermal conductivity, $\varepsilon$ : small scale parameter, $\operatorname{Pr}=\frac{\mu C_{p}}{k_{\infty}}$ : Prandtl number, $\theta_{w}=\frac{T_{w}}{T_{\infty}}:$ relative temperature ratio parameter, $A=\frac{U_{\infty}}{U_{0}}$ : ratio parameter, $M=\frac{\sigma B_{0}^{2} l}{\rho U_{0}}$ : magnetic field parameter, $L e=\frac{v}{D_{m}}$ : Lewis number, $K n=\frac{l k_{n}\left(C_{s}-C_{\infty}\right)^{n-1}}{U_{0}}:$ chemical reaction parameter. $T_{w}$ : surface temperature of cylinder and $T_{\infty}$ : ambient fluid temperature.

Skin friction coefficient, local Nusselt number and local Sherwood number can be defined as follows: 
$C_{f}=\frac{\tau_{w}}{\frac{1}{2} \rho\left(u_{w}\right)^{2}}$

$C f \operatorname{Re}_{x}^{1 / 2}=(1+K) f^{\prime \prime}(0)-K \frac{\bar{\beta}}{3}\left[f^{\prime \prime}(0)\right]^{3}$

$N u=\frac{x q_{w}}{k\left(T_{w}-T_{\infty}\right)}$

$N u \operatorname{Re}_{x}^{-1 / 2}=-\left(1+\frac{4}{3} R\right) \theta^{\prime}(0)$

$S h=\frac{x j_{w}}{D_{B}\left(C_{w}-C_{\infty}\right)}$

$\operatorname{Sh} \operatorname{Re}_{x}^{-1 / 2}=-\phi^{\prime}(0)$

where, $\operatorname{Re}_{x}=x u_{s} / v$ is the local Reynolds number.

Table 1. Comparison table of $-f$ "(0) for different values $M$ Comparison of $-f$ "(0) for different values $\mathrm{M}$ in the absence of the parameters $\mathrm{S}=\mathrm{A}=\mathrm{K}=\gamma=\lambda_{2}=0$

\begin{tabular}{|c|c|c|c|c|c|}
\hline $\mathrm{M}$ & $\begin{array}{c}\text { Anders } \\
\text { on et al. } \\
{[25]}\end{array}$ & $\begin{array}{c}\text { Prasad } \\
\text { et al. } \\
{[26]}\end{array}$ & $\begin{array}{c}\text { Mukhopa } \\
\text { dhyay et } \\
\text { al. } \\
{[27]}\end{array}$ & $\begin{array}{c}\text { Palani et } \\
\text { al } \\
{[28]}\end{array}$ & $\begin{array}{c}\text { Present } \\
\text { study }\end{array}$ \\
\hline 0.0 & 1.0000 & 1.0001 & 1.00017 & 1.0000 & 1.00000 \\
\hline 0.5 & 1.2249 & 1.2247 & 1.22475 & 1.2247 & 1.22474 \\
\hline 1 & 1.4140 & 1.4144 & 1.41445 & 1.4142 & 1.41421 \\
\hline 1.5 & 1.5810 & 1.5811 & 1.58114 & 1.5811 & 1.58113 \\
\hline 2 & 1.7320 & 1.7322 & 1.73220 & 1.7320 & 1.73205 \\
\hline
\end{tabular}

Table 2. Comparison table of $-\theta^{\prime}(0)$ for different values $\operatorname{Pr}$

\begin{tabular}{|c|c|c|c|c|c|c|}
\hline \multirow[t]{2}{*}{$\operatorname{Pr}$} & \multicolumn{6}{|c|}{$\begin{array}{l}\text { Comparison of }-\theta^{\prime}(0) \text { for different values } \operatorname{Pr} \text { in the } \\
\text { absence of the parameters } \mathrm{S}=\mathrm{A}=\mathrm{K}=\gamma=\lambda_{2}=\varepsilon=\mathrm{R}=\mathrm{Ec}= \\
\qquad \mathrm{Du}=\mathrm{A} * \mathrm{~B} *=0, \theta_{w}=1\end{array}$} \\
\hline & $\begin{array}{l}\text { Nadeem } \\
\text { et al } \\
{[30]}\end{array}$ & $\begin{array}{l}\text { Khan } \\
\text { et. al } \\
{[31]}\end{array}$ & $\begin{array}{l}\text { Golra } \\
\text { et. al } \\
{[31]}\end{array}$ & $\begin{array}{c}\text { Wang } \\
\text { [32] }\end{array}$ & $\begin{array}{l}\text { Naray } \\
\text { ana } \\
\text { et.al } \\
{[29]}\end{array}$ & $\begin{array}{l}\text { Presen } \\
\text { t study }\end{array}$ \\
\hline $\begin{array}{l}0 . \\
7 \\
\end{array}$ & 0.454 & 0.454 & 0.454 & 0.454 & $\begin{array}{c}0.453 \\
9 \\
\end{array}$ & $\begin{array}{c}0.454 \\
0 \\
\end{array}$ \\
\hline $\begin{array}{l}2 . \\
0\end{array}$ & 0.911 & 0.911 & 0.911 & 0.911 & $\begin{array}{c}0.911 \\
4\end{array}$ & $\begin{array}{c}0.911 \\
3\end{array}$ \\
\hline
\end{tabular}

\section{RESULTS AND DISCUSSION}

In this paper, calculations were carried out for different values of the parameters. The following default parameter values are adopted for computation: $\mathrm{A}=0.1, \lambda_{2}=0.1, \mathrm{n}=1, \mathrm{~S}=$ $0.5, \mathrm{~K}=0.1, \mathrm{Du}=0.1, \mathrm{R}=0.5, \varepsilon=0.1, \mathrm{Pr}=1.5, \gamma=0.1, \mathrm{Le}=5$, $\mathrm{Kn}=0.1, \theta_{w}=1.5, \mathrm{Su}=0.1, \mathrm{M}=0.5, \mathrm{~A}^{*}=0.1, \mathrm{~B}^{*}=0.1$ have been analyzed on axial $f^{\prime}, \theta$ and $\phi$ profiles. Figures (2-4) show the behavior of $\mathrm{M}$ on $f^{\prime}, \theta$ and $\phi$ profiles. Indicates that the dimensionless velocity $f^{\prime}$ is decreased and opposite behaviors show on $\theta$ and $\phi$ profiles as the value of $\mathrm{M}$ increases. Figures (5-7) represent the influence of $\mathrm{K}$ on $f^{\prime}, \theta$ and $\phi$ profiles. It is noticed that an increase in the value of $\mathrm{K}$, $f^{\prime}$ increases and $\theta$ and $\phi$ profiles decrease. With increases in the value of $\mathrm{A}, f^{\prime}$ profile increases whereas $\theta$ and $\phi$ profiles decrease, as shown in figures (8-10). Figures (11-13) show the $f^{\prime}, \theta$ and $\phi$ profiles against the similarity variable $\eta$ for various values of $\gamma$. We observe from these figures that the $f^{\prime}, \theta$ and $\phi$ profiles increase as $\gamma$ increases. Figures (14-15). An increase in the value of $\operatorname{Pr}$ is observed to decrease the $\theta$ and $\phi$ profiles. Prandtl number signifies the ratio between velocity diffusivity to energy diffusivity. Fluids with small value of Pr will possess large energy conductivities so that temperature can diffuse from the surface faster than for large value of Pr fluids. Figures (16-18) explain the effect of $\theta_{w}, \mathrm{R}, \varepsilon$ on $\theta$ profile. It is observed that, $\theta$ profile increases for increasing the values of $\theta_{w}, \mathrm{R}, \varepsilon$. This is due to the fact that an increase in radiation parameter provides more heat to fluid that causes an enhancement in the heat and thermal boundary layer thickness. Figure (19) shows the impact of Du on $\theta$ profile. An increase in the value of Du is observed to increase the $\theta$ profile. It has been experimentally verified that the diffusion of energy is caused by a composition gradient. This fact is known as the Dufour effect. Figures (20-21) show the impact of $\mathrm{Kn}$ and $\mathrm{Su}$ on $\phi$ profile. Rising the value of $\mathrm{Kn}$ and $\mathrm{Su}$ are observed to enhance $\phi$ profile. Kn increases the rate of interfacial mass transfer. Kn reduces the local concentration, thus increases its concentration gradient and its flux. Figures (22-23) show the impact of Le and $n$ on $\phi$ profile. An increase in the value of Le and $n$ are observed to suppress $\phi$ profile. It is due to the fact that Le is the ratio of velocity to mass diffusivities which means that when Le increases, mass diffusivity decreases and there is a reduction in concentration. Figures (24-25) display the effect of $\mathrm{A}^{*} \& \mathrm{~B}^{*}$ on $\theta$ profile. It is observed that, $\theta$ profile increases for increasing the values of $\mathrm{A}^{*} \& \mathrm{~B}^{*}$. Heat source parameters act like a heat producer, which gain the heat to the flow and enhances the temperature profiles. Table-1 Moreover, the present results for the skin friction coefficient $-f$ "(0) for different values of magnetic field parameter $M$ in the absence of the parameters $\mathrm{S}=\mathrm{A}=\mathrm{K}=\gamma=\lambda=0$ are compared with the available results of Anderson et al. [25], Prasad et al. [26], Mukhopadhyay et al. [27] and Palani et al [28]. . Table-2 Moreover, the present results for the Nusselt number $-\theta^{\prime}(0)$ for different values $\operatorname{Pr}$ in the absence of the parameters $\mathrm{S}=\mathrm{A}=\mathrm{K}=\gamma=\lambda_{2}=\varepsilon=\mathrm{R}=\mathrm{Ec}=$ $\mathrm{Du}=\mathrm{A} *=\mathrm{B} *=0, \theta_{w}=1$. Under some special conditions, present results have an excellent. Table- 3 for various values of the physical parameters, Namely A, Du, K, R, $\varepsilon, \operatorname{Pr}, \gamma$, Le, $\mathrm{Kn}, \theta_{w}, \mathrm{M}$ on $C_{f} \operatorname{Re}_{x}^{\frac{1}{2}}, N u \operatorname{Re}_{x}^{\frac{-1}{2}}$ and $S h \operatorname{Re}^{\frac{-1}{2}}$. From these tables, we noticed that with the increases in $\mathrm{M}, \mathrm{K}$, and $\gamma$ parameter the value of $C_{f} \operatorname{Re}_{x}^{\frac{1}{2}}$ decreases whereas increases in the value of $\mathrm{K} N u \operatorname{Re}_{x}{ }^{\frac{-1}{2}}$ and $S h \mathrm{Re}^{\frac{-1}{2}}$ increases. 


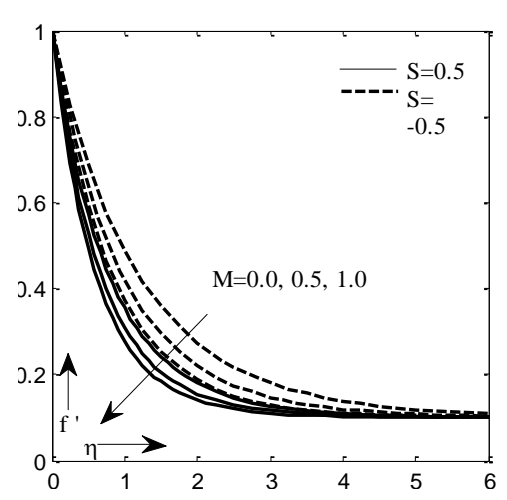

Figure 2. Impact of M on $f^{\prime}$

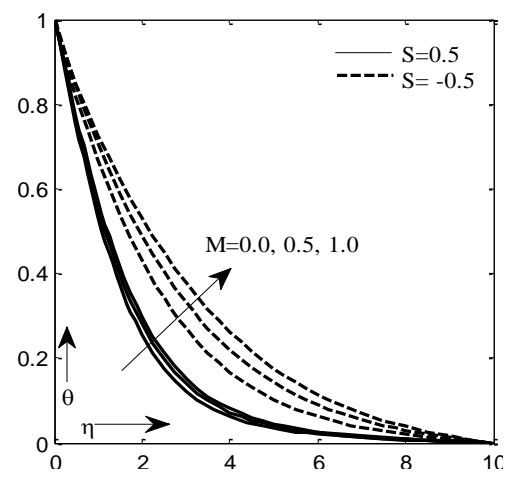

Figure 3.Impact of $\mathrm{M}$ on $\theta$

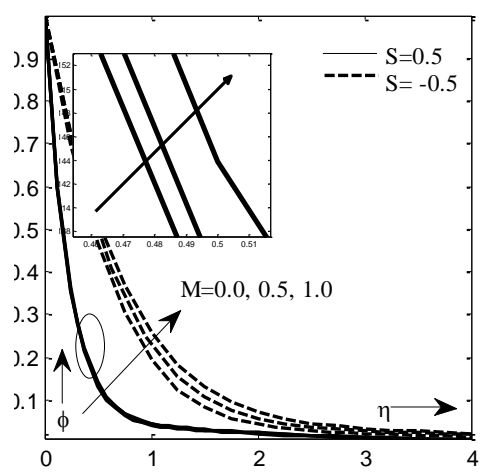

Figure 4. Impact of $M$ on $\phi$

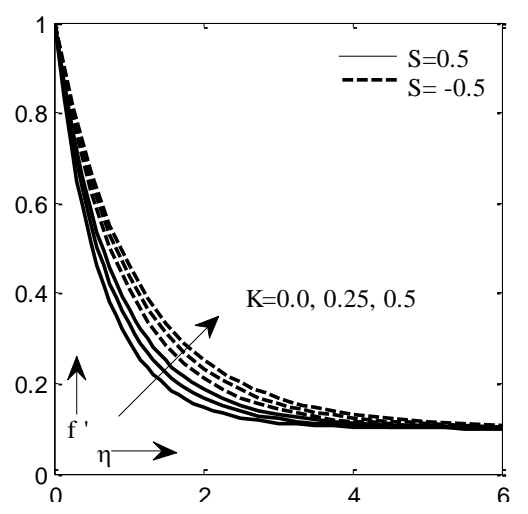

Figure 5. Impact of K on $f^{\prime}$

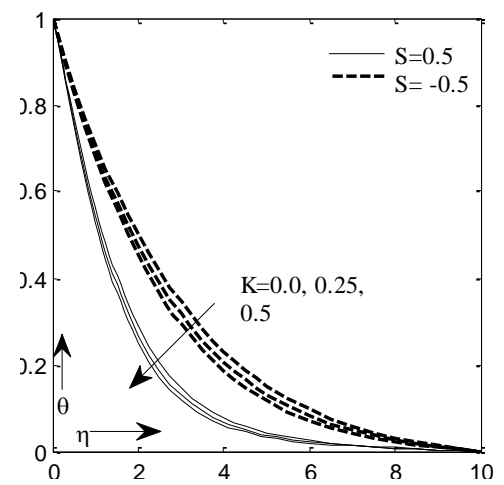

Figure.6 Impact of $\mathrm{K}$ on $\theta$

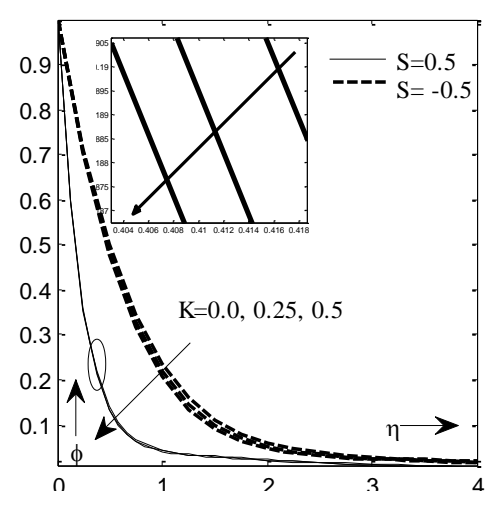

Figure 7. Impact of $\mathrm{K}$ on $\phi$

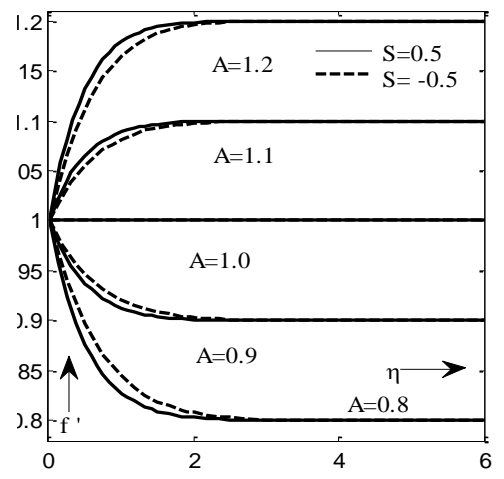

Figure 8. Impact of A on $f^{\prime}$

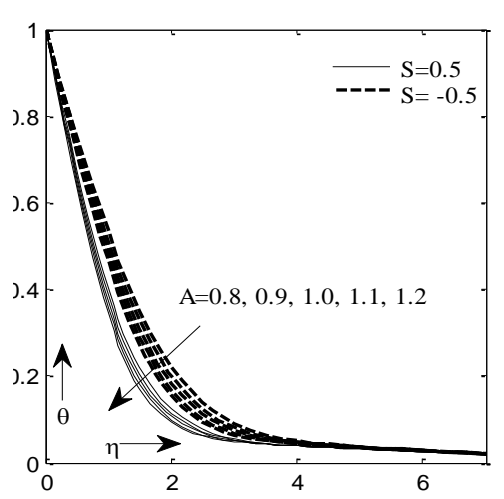

Figure 9. Impact of A on $\theta$ 


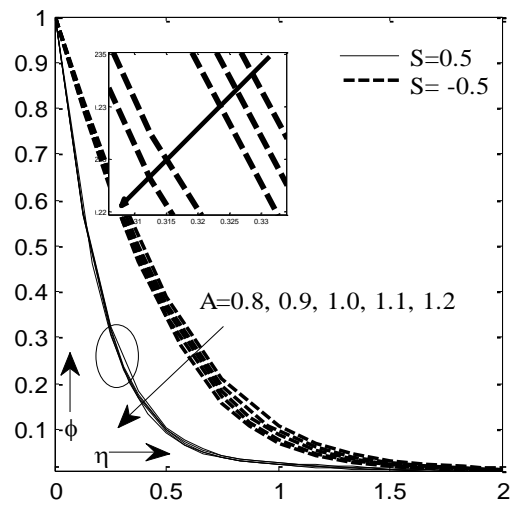

Figure 10. Impact of $\mathrm{A}$ on $\phi$

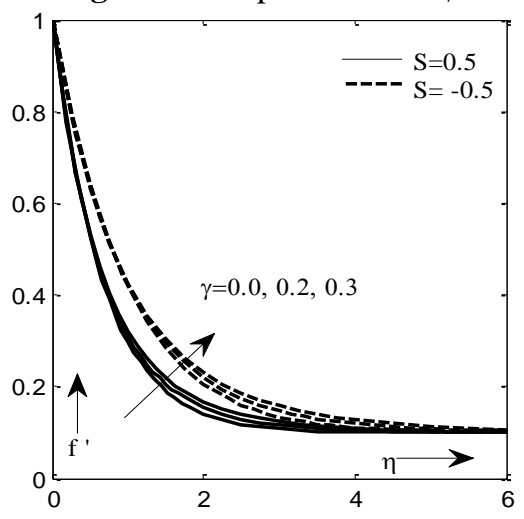

Figure 11. Impact of $\gamma$ on $f^{\prime}$

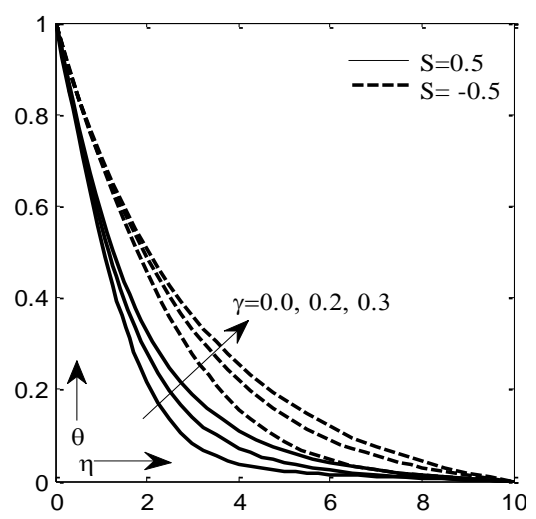

Figure 12. Impact of $\gamma$ on $\theta$

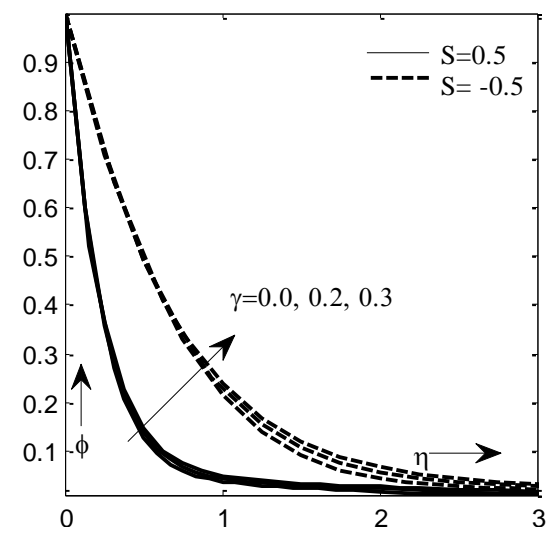

Figure 13. Impact of $\gamma$ on $\phi$

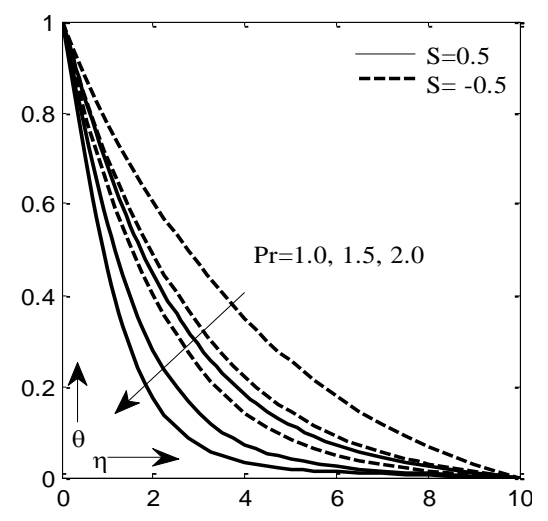

Figure 14. Impact of $\operatorname{Pr}$ on $\theta$

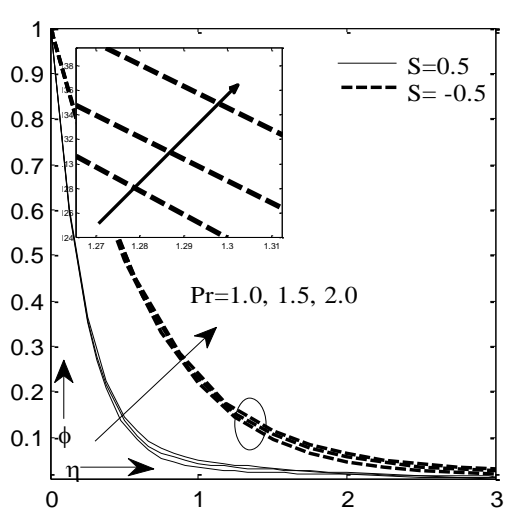

Figure 15. Impact of $\operatorname{Pr}$ on $\phi$

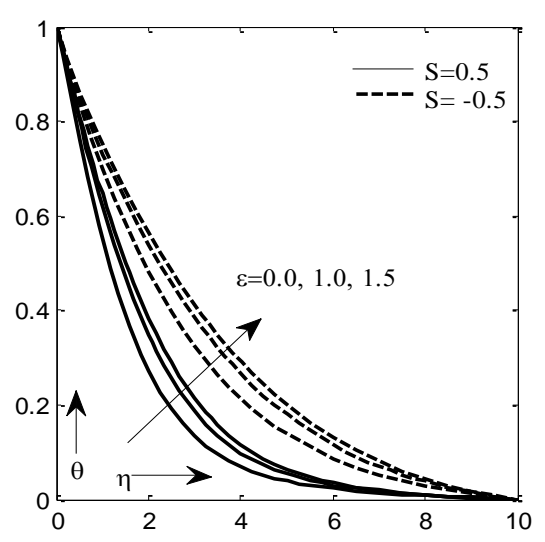

Figure 16. Impact of $\varepsilon$ on $\theta$

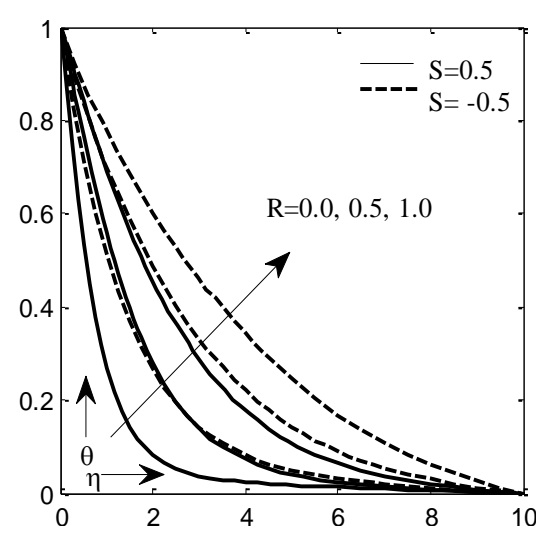

Figure 17. Impact of $\mathrm{R}$ on $\theta$ 


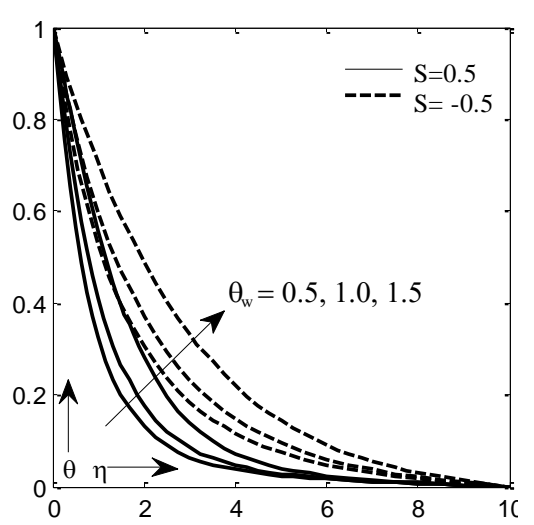

Figure 17. Impact of $\theta_{w}$ on $\theta$

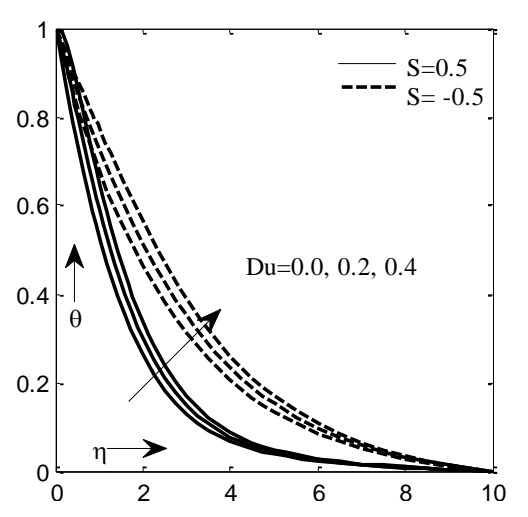

Figure 19. Impact of Du on $\theta$

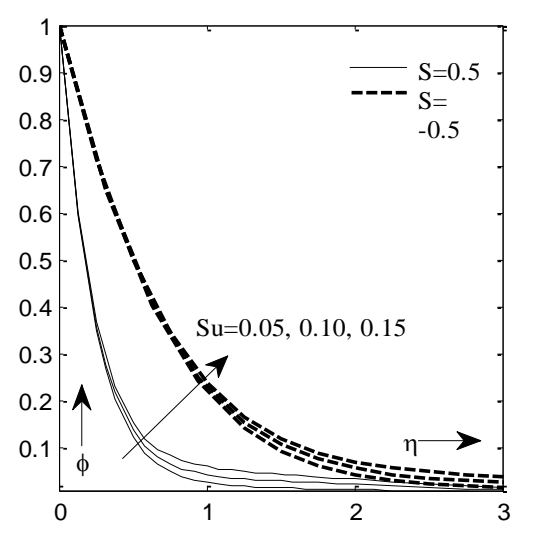

Figure 20. Impact of Su on $\phi$

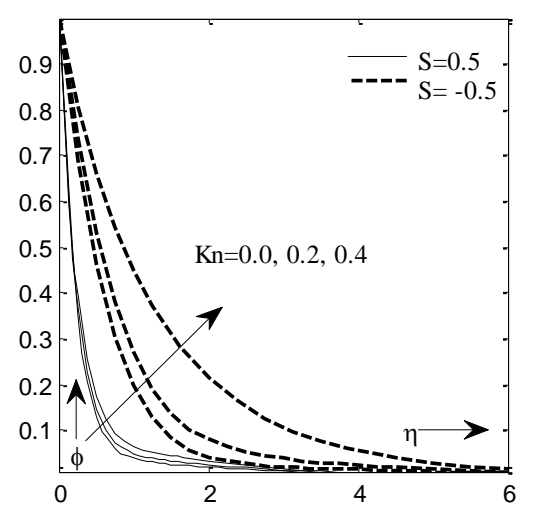

Figure 21. Impact of $\mathrm{Kn}$ on

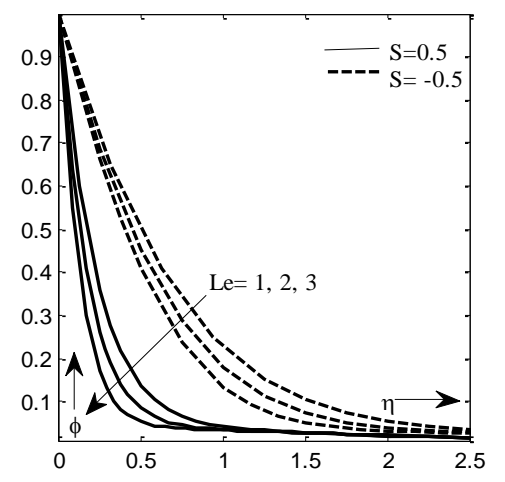

Figure 22. Impact of Le on $\phi$

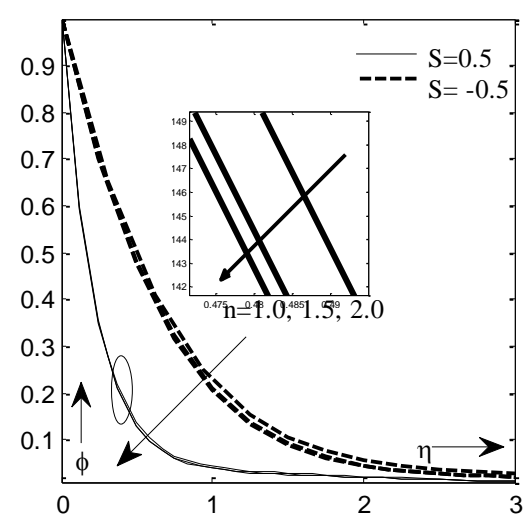

Figure 23. Impact of $n$ on $\phi$

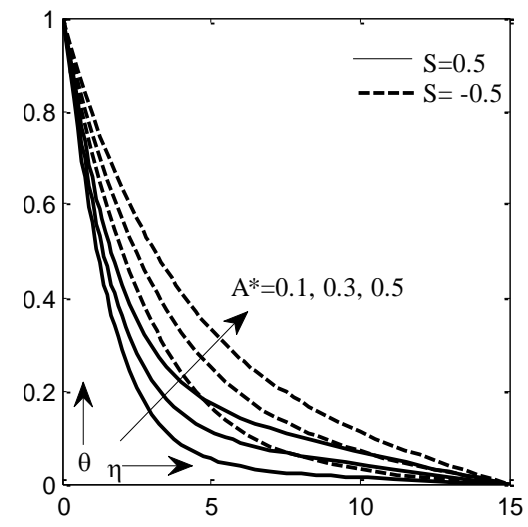

Figure 24. Impact of $\mathrm{A}^{*}$ on $\theta$

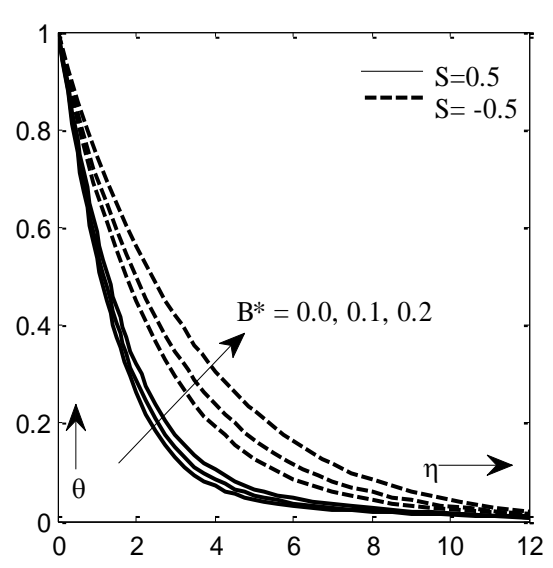

Figure 25. Impact of $\mathrm{B}^{*}$ on $\theta$ 
Table-3

\begin{tabular}{|c|c|c|c|c|c|c|c|c|c|c|c|c|}
\hline$\varepsilon \quad \mathrm{M}$ & $\mathrm{K}$ & $\gamma$ & Le & $\operatorname{Pr}$ & $\mathrm{R}$ & $\theta_{\mathrm{w}}$ & $\mathrm{A}$ & $\mathrm{Du}$ & $\mathrm{Kn}$ & - $C f \mathrm{Re}_{x}^{1 / 2}$ & $N u \operatorname{Re}_{x}^{-1 / 2}$ & $S h \operatorname{Re}_{x}^{-1 / 2}$ \\
\hline 0 & & & & & & & & & & 1.502219 & 0.760348 & 4.060689 \\
\hline 1 & & & & & & & & & & 1.502219 & 0.630711 & 4.079398 \\
\hline 2 & & & & & & & & & & 1.502219 & 0.544944 & 4.090668 \\
\hline 0 & & & & & & & & & & 1.305153 & 0.782987 & 4.099390 \\
\hline 1 & & & & & & & & & & 1.671311 & 0.714417 & 4.032911 \\
\hline 2 & & & & & & & & & & 1.958593 & 0.669742 & 3.983482 \\
\hline & 0.00 & & & & & & & & & 1.444668 & 0.729452 & 4.048704 \\
\hline & 0.25 & & & & & & & & & 1.584964 & 0.764166 & 4.081678 \\
\hline & 0.50 & & & & & & & & & 1.714564 & 0.791543 & 4.106555 \\
\hline & & 0.0 & & & & & & & & 1.462141 & 0.754909 & 4.048982 \\
\hline & & 0.2 & & & & & & & & 1.541780 & 0.737558 & 4.078769 \\
\hline & & 0.3 & & & & & & & & 1.580782 & 0.734518 & 4.096150 \\
\hline & & & 1 & & & & & & & 1.502228 & 0.946534 & 1.220979 \\
\hline & & & 2 & & & & & & & 1.502228 & 0.890381 & 2.035235 \\
\hline & & & 3 & & & & & & & 1.502220 & 0.839389 & 2.752226 \\
\hline & & & & 1.0 & & & & & & 1.502219 & 0.510620 & 4.095830 \\
\hline & & & & 1.5 & & & & & & 1.502219 & 0.744390 & 4.063113 \\
\hline & & & & 2.0 & & & & & & 1.502219 & 0.941818 & 4.033682 \\
\hline & & & & & 0.0 & & & & & 1.502219 & 1.064623 & 3.859308 \\
\hline & & & & & 0.5 & & & & & 1.502219 & 0.744390 & 4.063113 \\
\hline & & & & & 1.0 & & & & & 1.502219 & 0.698245 & 4.097192 \\
\hline & & & & & & 0.5 & & & & 1.502219 & 1.735235 & 3.857031 \\
\hline & & & & & & 1.0 & & & & 1.502219 & 1.256816 & 3.962370 \\
\hline & & & & & & 1.5 & & & & 1.502219 & 0.744390 & 4.063113 \\
\hline & & & & & & & 0.8 & & & 0.413809 & 1.026136 & 4.294352 \\
\hline & & & & & & & 1.0 & & & 0.000000 & 1.087361 & 4.359780 \\
\hline & & & & & & & 1.2 & & & 0.451286 & 1.144335 & 4.424770 \\
\hline & & & & & & & & 0.0 & & 1.502219 & 1.016664 & 3.997736 \\
\hline & & & & & & & & 0.2 & & 1.502219 & 0.461815 & 4.131562 \\
\hline & & & & & & & & 0.4 & & 1.502218 & 0.137283 & 4.278673 \\
\hline & & & & & & & & & 0.0 & 1.502219 & 0.736785 & 4.168052 \\
\hline & & & & & & & & & 0.2 & 1.502219 & 0.75252 & 3.950635 \\
\hline & & & & & & & & & 0.4 & 1.502219 & 0.771101 & 3.692596 \\
\hline
\end{tabular}

\section{CONCLUSION}

The present article includes the analysis of magneto-hydrodynamic (MHD) Powell-Eyring fluid flow in the presence of non-linear radiation, space dependent internal heat source and variable thermal conductivity over a permeable cylinder with suction/injection effects. We have considered Soret, Dufour and non-linear chemical reaction effect on heat and concentration equations. The effects of different physical key parameters such as magnetic parameter, suction parameter, thermal radiation parameter, and Prandtl number etc are plotted and discussed.

The conclusions of the present investigation are made as follows:

a) The results show that as the M increases the $f^{\prime}$ to the fluid suppresses the whereas opposite behavior is found for $\theta$ and $\phi$ profiles.

b) Increasing the value of $\mathrm{K}$ and $\mathrm{A}$ reduce the heat transfer coefficient between the cylinder surface and the fluid however, increasing following parameters, $\mathrm{R}, \gamma, \mathrm{A}^{*}, \mathrm{~B}^{*}, \mathrm{Ec}$, $M$ increases it. c) Increasing the value of K, A, Le, $n$ suppress $\phi$ profile whereas increases the value of $\mathrm{M}, \mathrm{Kn}, \mathrm{Su}, \mathrm{Pr}, \gamma$ enhances the $\phi$ profile.

d) Increasing the value of $\mathrm{M}, \mathrm{K}$, and $\gamma$ parameters, decreases the value of $C_{f} \operatorname{Re}_{x}^{\frac{1}{2}}$

\section{REFERENCES}

[1] Khan MI, Kiyani MZ, Malik MY, Yasmeen T, Khan MWA, Abbas T. (2016). Numerical investigation of magneto hydrodynamic stagnation point flow with variable properties. Alexandria Engineering Journal 55(3): 2367-2373.

[2] Krishna PM, Sandeep N, Reddy JVR, Sugunamma V. (2016). Dual solutions for unsteady flow of powelleyring fluid past an inclined stretching sheet. J. of Naval Arch. and Marine Engg. https:// doi.org/ 10.3329/jname. v13i1.25338. 
[3] Mahanthesh B, Gireesha BJ, Gorla RSR. (2017). Unsteady three-dimensional MHD flow of a nano Eyring-Powell fluid past a convectively heated stretching sheet in the presence of thermal radiation, viscous dissipation and Joule heating. J. of the Association of Arab Universities for Basic and Applied Sciences 23: 7584.

[4] Akbar NS, Ebaid A. (2015) Numerical analysis of magnetic field on eyring-powell fluid flow towards a stretching sheet. Journal of Magnetism and Magnetic Materials 382: 355-358.

[5] Javed T, Ali N, Abbas Z, Sajid M. (2013). Flow of an Eyring Powell non-Newtonian fluid over a stretching sheet. Chem. Eng. Comm. 200 (3): 327-336.

[6] Hayat T, Gull N, Farooq M, Alsaedi A. (2016) Thermal radiation effect in MHD flow of Powell-Eyring nanofluid induced by a stretching cylinder J Aerosp. Eng. 29 (1): 04015011-13.

[7] Hayat T, Ashraf MB, Shehzad SA, Abouelmagd E. (2015). Three-dimensional flow of Eyring Powell nanofluid over an exponentially stretching sheet. Int. J. Numer. Methods Heat Fluid Flow 25(3): 593-616.

[8] Hayat T, Pakdemirli M, Aksoy T. (2013). Similarity solutions for boundary layer equations of a Powel-Eyring fluid. Mathematical and Computational Applications 18(1): 62-70.

[9] Hayat T, Waqas M, Shehzad SA, Alsaedi A. (2016). Mixed convection stagnation-point flow of Powell Eyring fluid with Newtonian heating, Thermal Radiation, and Heat Generation/Absorption. https:// doi.org/ 10.1061/(ASCE)AS.1943-5525.0000674.

[10] Hayat T, Makhdoom S, Awais M, Saleem S, Rashidi M M (2016). Axisymmetric Powell-Eyring fluid flow with convective boundary condition: optimal analysis. Appl. Math. Mech. -Engl. Ed. https:// doi.org/ 10.1007/s10483016-2093-9.

[11] Gaffar SA, Prasad VR, Reddy EK. (2016). MHD free convection flow of Eyring-Powell fluid from vertical surface in porous media with Hall/ionslip currents and ohmic dissipation. Alexandria Engineering Journal 55(2): 875-905.

[12] Madhu M, Kishan N. (2016). Finite element analysis of heat and mass transfer by MHD mixed convection stagnation-point flow of a non-Newtonian power-law nano fluid towards a stretching surface with radiation. J. of the Egyptian Mathematical Society 24: 458-470.

[13] Makinde OD. (2012). Heat and mass transfer by MHD mixed convection stagnation point flow toward a vertical plate embedded in a highly porous medium with radiation and internal heat generation. Meccanica 47: 1173-84.

[14] Jain S, Kumar V, Bohra S. (2017). Entropy generation for MHD radiative compressible fluid flow in a channel partially filled with porous medium, Global and Stochastic Analysis, SI., 13-31.

[15] Jain S, Choudhary R. (2017). Soret and Dufour effects on MHD fluid flow due to moving permeable cylinder with radiation. Global and Stochastic Analysis, SI, pp. 75-84.

[16] Jain S. (2006) Temperature distribution in a viscous fluid flow through a channel bounded by a porous medium and a stretching sheet, J. Rajasthan Acad. Phy. Sci. 4: 477482.

[17] Jain S, Choudhary R. (2015) Effects of MHD on boundary layer flow in porous medium due to exponentially shrinking sheet with Slip, Procedia Engg. 127: 1203-1210.

[18] Jain S, Parmar A. (2017). Comparative study of flow and heat transfer behavior of Newtonian and non-Newtonian fluids over a permeable stretching surface. Global and Stochastic Analysis SI: 41-50.

[19] Das K, Sharma RP, Sarkar A. (2016). Heat and mass transfer of a second-grade magneto-hydrodynamic fluid over a convectively heated stretching sheet. J. of Computational Design and Engg. 3(4): 330-336.

[20] Chauhan DS, Rastogi P. (2011). Heat transfer and entropy generation in MHD flow through a porous medium past a stretching sheet. Inter. J. of Energy \& Tech. 3(15): 1-13.

[21] Chauhan DS, Rastogi P. (2012). Unsteady MHD flow and heat transfer through a porous medium past a nonisothermal stretching sheet with slip conditions. International Journal of Energy and Technology 4(17): 111.

[22] Chauhan DS, Kumar V. (2012). Radiation effects on unsteady flow through a porous medium channel with velocity and temperature slip boundary conditions, Applied Mathematical Sciences 6(36): 1759-1769.

[23] Ska Md T, Das K, Kundu PK, (2016). Effect of magnetic field on slip flow of nanofluid induced by a non-linear permeable stretching surface. Applied Thermal Engineering 104: 758-766.

[24] Animasaun IL, Adebile EA, Fagbade AI. (2016). Casson fluid flow with variable thermo-physical property along exponentially stretching sheet with suction and exponentially decaying internal heat generation using the homotopy analysis method, J. of the Nigerian Math. Society 35: 1-17.

[25] Andersson HI, Hansen OR, Holmedal B. (1994). Diffusion of a chem-ically reactive species from a stretching sheet. Int J Heat Mass Transfer 37: 659-64.

[26] Prasad KV, Sujatha A, Vajravelu K, Pop I. (2012). MHD flow and heat transfer of a UCM fluid over a stretching surface with variable thermos-physical properties. Meccanica 47: 1425-39.

[27] Mukhopadhyay S, Golam AM, Wazed AP. (2013). Effects of transpiration on unsteady MHD flow of an UCM fluid passing through a stretching surface in the presence of a first order chemical reaction. Chin Phys B 22: 124701.

[28] Palani S, Kumar BR, Kameswaran PK. (2016) Unsteady MHD flow of an UCM fluid over a stretching surface with higher order chemical reaction, Ain Shams Engg. J. 7: 399-408.

[29] Narayana KL, Gangadhar K, Subhakar MJ. (2015). Effect of viscous dissipation on heat transfer of magnetoWilliamson Nano fluid, IOSR-JM 11(4): 25-37.

[30] Nadeem S, Hussain ST. (2013). Flow and heat transfer analysis of Williamson Nanofluid, Appl Nanosci. Einstein, Ann. Phys. 19: 286.

[31] Khan WA, Pop I. (2010). Boundary-layer flow of a Nanofluid past a stretching sheet, Int. J. Heat Mass Transf. 53: 2477-2483.

[32] Gorla RSR, Sidawi I. (1994). Free convection on a vertical stretching surface with suction and blowing, Appl. Sci Res. 52: 247-257.

[33] Wang Y. (1989). Free convection on a vertical stretching surface, J Appl. Math Mech. 69: 418-420. 


\section{NOMENCLATURE}

K

$R$

$S$

Du

$\mathrm{Su}$

$k_{\infty}$

$\operatorname{Pr}$

A

$M$ material fluid parameters

radiation parameter

suction /injection parameter

Dufour number

Soret number

thermal conductivity

Prandtl number

ratio parameter

magnetic field parameter

Lewis number
Le chemical reaction

$\mathrm{Kn}$

\section{Greek symbols}

$\theta$

$\phi$

$\lambda$

$\gamma$

$\varepsilon$

$\theta_{w}$
Dimensionless temperature

Dimensionless concentration.

fluid parameter

curvature parameter small scale parameter

relative temperature ratio parameter 